\title{
HEMATOMA ETMOIDAL PROGRESSIVO EM EQUINO REVISÃO DE LITERATURA E RELATO DE CASO
}

\author{
PROGRESSIVE ETHMOID HEMATOMA IN HORSES \\ REVIEW AND CASE REPORT
}

\author{
G. M. BUENO ${ }^{1}$, N. S. BERNARDI ${ }^{1}$, R. CAMPOS $^{2}$, F. M. CAMPOS ${ }^{2}$, \\ J. C. LACERDA-NETO ${ }^{1}$, D. P. M. DIAS ${ }^{*}$
}

\begin{abstract}
RESUMO
O hematoma etmoidal progressivo (HEP) trata-se de uma massa não neoplásica que se expande a partir de hemorragias sucessivas da camada submucosa do labirinto etmoidal ou dos seios paranasais. Técnicas de diagnóstico, como endoscopia das vias aéreas superiores, radiografia e tomografia computadorizada, visam determinar a extensão das lesões. O tratamento é preconizado de acordo com o tamanho e localização do HEP, além da disponibilidade de equipamentos. As opções de tratamento incluem a ressecção cirúrgica, redução da massa por aplicação de agentes criogênicos ou laser e, a injeção de formaldeído intralesional. O prognóstico a longo prazo é reservado a desfavorável devido a alta taxa de recidivas da lesão. Este artigo fornece uma visão geral sobre a doença e descreve um caso de HEP tratado com sucesso pelo uso de injeção de formaldeído intralesional.
\end{abstract}

PALAVRAS-CHAVE: endoscópio, equino, etmóide, formaldeído, seio paranasal.

\section{SUMMARY}

Progressive ethmoid hematoma (PEH) is a non-neoplastic mass which expands following repeated hemorrhage in the submucosa of the ethmoidal labyrinth or paranasal sinuses. Diagnostic techniques, as upper airway endoscopy, radiography and computed tomography, aims to determine the extent of the lesions. Treatment is based on size and location of the PEH, along with consideration of equipment availability. Treatment options include surgical resection, mass reduction with application of cryogens or laser, and intralesional formaldehyde injection. Prognosis for long-term resolution remains guarded to poor due to high rates of recurrence. This article provides an overview of the disease and reports a case of $\mathrm{PEH}$ successfully treated by the use of intralesional formaldehyde injection.

KEY-WORDS: endoscope, equine, ethmoid, formaldehyde, paranasal sinus.

\footnotetext{
${ }^{1}$ Faculdade de Ciências Agrárias e Veterinárias (FCAV), Unesp, Jaboticabal - SP. * Autor para correspondência: Deborah Penteado Martins Dias, Departamento de Clínica e Cirurgia Veterinária, FCAV/Unesp, Via de acesso Prof. Paulo Donato Castellane, s/n Jaboticabal, SP, 14884-900, Brasil. E-mail: dpmdias@fcav.unesp.br, deborah_dias@hotmail.com

${ }^{2}$ Equine Center, Jockey Club, São Paulo - SP.
} 


\section{INTRODUÇÃO E ETIOPATOGENIA}

O hematoma etmoidal progressivo (HEP) em equinos foi descrito por Cook \& Littlewort em 1974. Trata-se de uma massa não neoplásica, angiomatosa e encapsulada, que se desenvolve no labirinto etmoidal ou a partir dos seios paranasais, mais comumente o maxilar e o esfenopalatino (COOK \& LITTLEWORT, 1974; SULLIVAN et al., 1984; TATE, 2002). Em estudos restrospectivos com casuísticas hospitalares, a prevalência foi de 1 caso de HEP para cada 2500 equinos referidos para atendimento hospitalar (BELL et al., 1993a).

A etiologia que leva ao desenvolvimento do HEP é desconhecida, entretanto sabe-se que ocorrem hemorragias sucessivas que se iniciam a partir da camada submucosa do trato respiratório superior. A massa cresce lentamente, porém de forma progressiva, causando estiramento e espessamento da camada mucosa que a recobre formando uma pseudo-cápsula fibrosa (COOK \& LITTLEWORT, 1974; CONTI et al., 2003). Com a evolução da lesão, há destruição do epitélio respiratório local e necrose óssea por compressão (COOK \& LITTLEWORT, 1974). A origem do sangramento determina 4 diferentes formas de HEP, sendo a primeira de origem no seio maxilar (Tipo 1), a segunda de origem no seio esfenopalatino (Tipo 2), a terceira de origem nos próprios cornetos etmoidais (Tipo 3) e a quarta, extremamente rara, de origem em qualquer outra região excluindo as já citadas, como por exemplo o seio frontal (Tipo 4). As lesões de origem no seio maxilar ou esfenopalatino podem preencher completamente o seio acometido e, invadir ou não a cavidade nasal. A lesão de origem nos cornetos etmoidais, usualmente se protrui para o interior da cavidade nasal (TATE, 2002).

O HEP é usualmente único e unilateral, sendo a apresentação bilateral atribuída à expansão da lesão inicialmente unilateral e de origem nos cornetos etmoidais, através do septo etmoidal (HEAD \& DIXON, 1999; STICH et al., 2001). Macroscopicamente, a lesão apresenta formato lobulado e superfície lisa, com coloração variando de vermelho-púrpura a amareloesverdeado (HEAD \& DIXON, 1999; CONTI et al., 2003). Quanto às dimensões, HEP de origem no seio maxilar (Tipo 1) tendem a ser menores que os de origem no labirinto etmoidal (Tipo 3) (COOK \& LITTLEWORT, 1974; SULLIVAN et al., 1984), podendo atingir até 12 $\mathrm{cm}$ de diâmetro (TREMAINE et al., 1999). Histologicamente, por se tratar de tecido do trato respiratório superior, a massa é recoberta por epitélio pseudoestratificado colunar ciliado contendo glândulas e tecido conjuntivo denso. Em algumas áreas observa-se epitélio escamoso estratificado apresentando ulcerações e infiltrado inflamatório. O tecido mais profundo (estroma) contém sangue, tecido conjuntivo denso, infiltrado de plasmócitos, linfócitos e ocasionalmente neutrófilos (TREMAINE et al., 1999). Os pequenos vasos observados em grande quantidade, apresentam depósitos de calcáreo na parede vascular (PLATT, 1975). As áreas de hemorragia levam ao acúmulo de macrófagos e células multinucleadas contendo hemossiderina (HEAD \&
DIXON, 1999). Assim, a coloração varia de vermelho escuro a amarelo acinzentado de acordo com o tipo e distribuição dos pigmentos de hemoglobina após os episódios mais recentes de sangramento intralesional (TREMAINE et al., 1999).

\section{SINAIS CLÍNICOS}

Independentemente da forma, o sinal clínico característico é a epistaxe de forma intermitente e unilateral (BELL et al., 1993a), que ocorre a partir de ulcerações na superfície da cápsula do HEP (TREMAINE et al., 1999). A epistaxe bilateral é descrita para os casos onde a lesão tem origem nos cornetos etmoidais de um dos lados e invade o contralateral (HEAD \& DIXON, 1999). Outros sinais clínicos descritos incluem chacoalhar de cabeça, dispnéia, tosse e expiração de odor fétido (COOK \& LITTLEWORT, 1974; ETHERINGTON et al., 1982; SCHUMACHER et al., 1998; TATE, 2002). Devido à diminuição da área para passagem do ar pela cavidade nasal do lado acometido, o equino pode apresentar ruído ventilatório anormal que se torna mais pronunciado durante o exercício (SPECHT et al., 1990; FREEMAN, 2003). Observa-se secreção mucopurulenta em alguns casos e, raramente deformação da face (BELL et al., 1993a). Segundo Colbourne et al. (1997), a distorção facial secundária ao HEP ocorre mais comumente em potros devido ao crescimento ósseo ainda presente.

\section{DIAGNÓSTICO}

A determinação do diagnóstico se inicia pelo histórico de epistaxe e se conclui por meio de exames de imagem (COOK \& LITTLEWORT, 1974). O exame endoscópico da cavidade nasal permite a visibilização direta da massa no interior da cavidade nasal, localizada no aspecto caudal dos cornetos etmoidais ou, identifica um traço de sangue localizado no meato nasal ou na abertura nasomaxilar, proveniente de massas menores ou localizadas nos seios paranasais (TATE, 2002; WAGUESPACK \& TAINTOR, 2011). Entretanto, o exame endoscópico da cavidade nasal não é capaz de determinar a extensão da lesão.

O acometimento dos seios paranasais é verificado por meio de exame radiográfico quando observa-se aumento de opacidade caracterizando a presença de tecido mole no interior do seio (GIBBS \& LANE, 1987; GASSER et al., 2000). Indica-se a realização do exame nas projeções lateral, dorsoventral e latero-dorsal oblíqua a $30^{\circ}$ (FEICHTENHOFER et al., 2013).

A tomografia computadorizada também identifica lesões no interior dos seios paranasais, sendo este o único método que permite a identificação precisa dos limites anatômicos da massa determinando sua exata distribuição espacial (TEXTOR et al., 2012). A tomografia apresenta a vantagem de formar uma imagem em secção transversal sem a sobreposição de outras estruturas anatômicas, o que ocorre no exame radiográfico (SOLANO \& BRAWER, 2004; WAGUESPACK \& TAINTOR, 2011; NICKELS, 
2012). Com imagens de tomografia computadorizada, hematomas etmoidais aparecem como massas de tecido mole bem definidas, homogêneas ou heterogêneas, localizadas nos turbinados etmoidais ou no interior seios paranasais (PORTER \& WERPY, 2014). A desvantagem da tomografia é a necessidade de anestesia geral para sua realização (WAGUESPACK \& TAINTOR, 2011).

Caso a radiografia indique acometimento de um dos seios paranasais e a tomografia computadorizada não esteja disponível, o HEP pode ser diagnosticado por meio da realização de trepanação do seio seguida de sinoscopia (CONTI et al., 2003). Independente da localização, o diagnóstico só pode ser confirmado após o exame histopatológico da lesão (GASSER et al., 2000; TREMAINE \& DIXON, 2001).

O diagnóstico diferencial do HEP inclui todas as afecções que possam promover epistaxe persistente ou intermitente, tais como lesões por corpos estranhos de estruturas associadas à cavidade nasal, rinite fúngica ou ulcerativa, neoplasias, fraturas de crânio, micose de bolsa gutural, abscessos pulmonares e pleuropneumonia (NICKELS, 2012).

\section{TRATAMENTO}

Devido à diminuição progressiva da área para passagem do ar pela cavidade nasal, podendo levar à obstrução unilateral completa, o tratamento conservativo não é indicado (GASSER et al., 2000). A excisão cirúrgica do HEP com acesso por trepanação dos seios frontal ou maxilar foi o primeiro tratamento proposto (COOK \& LITTLEWORT, 1974), tendo sido realizada por vários anos. Boulton (1985) descreveu a remoção de massas com origem no etmóide invadindo a cavidade nasal por acesso através da criação de um "flap" no osso nasal.

Para o sucesso do tratamento cirúrgico o tecido de origem do HEP deve ser inteiramente removido. Entretanto, o difícil acesso à origem de massas localizadas no seio esfenopalatino e no labirinto etmoidal podem resultar em excisão incompleta. Ademais, a hemorragia intraoperatória profusa devido ao vasto leito vascular dificultam o procedimento (FREEMAN, 2003; AHERN \& PARENTE, 2008). A excisão cirúrgica em posição quadrupedal se torna ainda mais difícil, havendo a necessidade de anestesia geral para a maioria dos casos (AHERN \& PARENTE, 2008).

A complicação mais comum após a excisão cirúrgica é a recidiva da lesão (HEAD \& DIXON, 1999; AHERN \& PARENTE, 2008). Outras complicações incluem encefalites, deiscência da sutura, periostites (SCHUMACHER et al., 1998) e trauma a estruturas relacionadas anatomicamente ao encéfalo lesão cerebral após o procedimento (TREMAINE, 1999).

A remoção do HEP por criocirurgia demonstrou-se efetiva com as vantagens de poder ser realizada por via nasal sob visibilização endoscópica, com o animal em posição quadrupedal e sedado. A técnica propõe a remoção sequencial de pequenos fragmentos da massa após o congelamento com nitrogênio líquido. O risco de hemorragia é mínimo e a origem do HEP é destruída, diminuindo a recorrência da lesão. Entretanto, a criocirurgia não é efetiva na regressão de lesões extensas e há risco de erosão da placa cribiforme e consequente dano cerebral (BELL et al., 1993b).

Outro método de tratamento é fotoablação com o uso do neodymium:yttrium aluminum garnet (Nd:YAG), laser de 100W. O calor do feixe do laser causa coagulação do o tecido lesionado do trato respiratório à temperatura de $60^{\circ} \mathrm{C}$ e vaporização quando a lesão é aquecida a $100^{\circ} \mathrm{C}$ (PALMER, 2003). $\mathrm{Na}$ cirurgia com uso do laser Nd:YAG, o HEP pode ser acessado via nasal ou através de sinusotomia, dependendo da localização da lesão (ORSINI, 2002), apresentando a mesma vantagem da criocirurgia de possibilidade de realização em posição quadrupedal com o animal sob sedação (ROTHAUG \& TULLENERS, 1999). Há o risco de complicações devido a danos térmicos aos tecidos adjacentes à massa que recebeu disparos do laser (AHERN \& PARENTE, 2008) e o alto custo do equipamento é fator limitante para o seu uso (TATE, 1991). A taxa de sucesso foi de $70 \%$ em 41 equinos apresentando HEP e tratados por fotoablação com o laser Nd:YAG, não havendo sinais de recidiva (TATE, 2002).

Tanto a crioterapia quanto a irridiação de laser podem ser aplicadas em associação à excisão cirúrgica visando conter a hemorragia e destruir tecido residual do HEP (PALMER, 2003).

Considerando as complicações relativas aos demais procedimentos descritos, Schumacher et al. (1998), propuseram como terapia a ablação química por injeção intralesional seriada de solução de formaldeído a $4 \%$ por via transendoscópica, obtendo regressão completa da massa e remissão dos sinais clínicos em 17 de 27 lesões tratadas. A injeção de formaldeído tem como ação a hidrólise de proteínas, promovendo a desidratação tecidual e coagulação da lesão (SCHUMACHER et al. 1998). A solução deve ser injetada por um cateter de polipropileno com agulha retrátil de $23 \mathrm{GA}$, inserido pelo canal de biópsia do endoscópio, com o animal em posição quadrupedal e sedado. O procedimento é monitorado visualmente pelo endoscópio e, em relação ao volume administrado, o HEP deve ser preeenchido até ingurgitar e a solução de formaldeído começar a extravasar ao redor do cateter (SCHUMACHER et al., 1998). Seguindo esta técnica, o extravasamento da solução de formaldeído sobre o epitélio respiratório adjacente é mínimo e causa somente inflamação local (CONTI et al., 2003).

Recomenda-se o tratamento seriado, respeitando-se o intervalo de 3 a 4 semanas entre as aplicações de formaldeído, até que a lesão desapareça ou se reduza ao ponto de não permitir mais a injeção (SCHUMACHER et al., 1998). A partir da ablação química proposta por Schumacher et al. em 1998, outros autores relataram o sucesso deste tratamento (MARRIOTT et al., 1999; CONTI et al., 2003). Nogueira et al. (2007), descreveram um caso tratado até completa resolução com o uso de formaldeído a 4\%. Entretanto, o HEP se encontrava no interior do seio maxilar e a administração foi realizada via trepanação temporária do seio.

$\mathrm{O}$ tratamento intralesional com formaldeído tem a vantagem de ser um procedimento relativamente 
barato e seguro. As complicações são raras e incluem o desenvolvimento de sinusite severa, necrose das conchas nasais (FREEMAN, 2003) e lesão do sistema nervoso central (FREES et al., 2001). Segundo Frees et al. (2001), no relato do tratamento de HEP em um equino de 18 anos, o uso da solução de formaldeído na concentração de $4 \%$ levou a quadro neurológico de ataxia, alienação e "head pressing". Neste caso, o HEP levou a erosão e necrose da placa cribiforme ventral, promovendo o contato da solução de formaldeído injetada com o bulbo olfatório e lobo cerebral frontal.

Independentemente do tratamento proposto, avaliações endoscópicas da cavidade nasal devem ser realizadas a cada 6 meses, visto que a ausência de sinais clínicos não determina a ausência de recidivas da lesão. Além disso, a identificação precoce permite que a lesão seja tratada enquanto é pequena (FREEMAN, 2003). Mesmo com a abordagem terapêutica adequada, o prognóstico é considerado reservado a desfavorável devido a alta taxa de recidivas, que pode chegar a $44 \%$ (SULLIVAN et al., 1984).

Orsini \& Hausner (2004), identificaram a expressão do fator de crescimento endotelial vascular no HEP, sugerindo que inibidores da angiogênese podem ser efetivos no tratamento da lesão, principalmente ao evitar recidivas após a remoção cirúrgica.

Apesar de amplamente descrito, o HEP ainda é considerado uma afecção rara em equinos, havendo poucos relatos da enfermidade no Brasil. Assim, descreve-se um caso de HEP tratado com sucesso por ablação química com solução de formaldeído.

\section{RELATO DO CASO}

Uma égua da raça Puro Sangue Árabe, com 13 anos de idade e $390 \mathrm{Kg}$, utilizada na modalidade enduro equestre, foi encaminhada para atendimento Veterinário com queixa de apresentar ruído ventilatório anormal, dispnéia exacerbada quando em exercício e presença de secreção sanguinolenta proveniente das cavidades nasais direita e esquerda. Ao exame físico inicial, observou-se frequência cardíaca de 36 batimento por minuto, frequência respiratória de 16 movimentos respiratórios por minuto, mucosas róseas, tempo de preenchimento capilar de 2 segundos e temperatura retal de $37,9^{\circ} \mathrm{C}$.

A partir do histórico, indicou-se exame endoscópico das vias aéreas superiores que revelou uma massa de forma oval de aproximadamente $5 \mathrm{~cm}$ de comprimento por $3,5 \mathrm{~cm}$ de largura, localizada na porção caudal da cavidade nasal esquerda (Figura 1A). A lesão de coloração amarelada e superfície lisa e brilhante, encontrava-se relacionada aos cornetos etmoidais. Observou-se ainda porção do HEP invadindo a cavidade nasal direita, com aproximadamente $3 \mathrm{~cm}$ de diâmetro, igualmente relacionada aos cornetos etmoidais e identificada pela avaliação endoscópica (Figura 1B). A lesão localizada na cavidade esquerda apresentava mobilidade aos movimentos de inspiração e expiração. Os sinais clínicos apresentados em associação com a característica macroscópica da lesão orientou a conclusão do diagnóstico em HEP. Indicou-se como tratamento a ablação química pela administração intralesional transendoscópica de solução de formaldeído a $1,5 \%^{3}$.

Previamente à ablação química, realizou-se traqueostomia no terço médio da região cervical ventral. Para tanto, o animal foi contido em tronco, sedado com $0,01 \mathrm{mg} / \mathrm{kg}$ de cloridrato de detomidina ${ }^{4}$ por via intravenosa, quando realizou-se anestesia no local da intervenção com $10 \mathrm{~mL}$ de cloridrato de lidocaína a $2 \%$ sem vasoconstritor ${ }^{5}$. Verificada a eficácia do procedimento anestésico e, após preparação asséptica da região cervical ventral, o procedimento cirúrgico foi iniciado por incisão de pele de $8 \mathrm{~cm}$ de comprimento, seguida de divulsão do tecido subcutâneo e divisão dos feixes do músculo esternotireóideo em posição mediana. Identificou-se dois anéis traqueais e realizou-se fenestração da traquéia por excisão da porção ventral incluindo a metade da largura de cada anel. Finalizada a fenestração, introduziu-se traqueotubo metálico que foi suturado à pele por pontos simples separados com nylon $\mathrm{n}^{\mathrm{o}} 1$.

Por visibilização endoscópica e uso do canal auxiliar de biópsia, injetou-se $20 \mathrm{~mL}$ de cloridrato de lidocaína a $2 \%$ sem vasoconstritor nas estruturas adjacentes ao HEP. Após o bloqueio anestésico e, ainda através do canal de biópsia, administrou-se 15 $\mathrm{mL}$ de solução de formaldeído a $1,5 \%$ no interior da lesão pelo lado esquerdo em diferentes pontos (Figura 1C). Prescreveu-se associação de penicilina potássica, procaína e benzatina $^{6}$ na dose de $30000 \mathrm{UI} / \mathrm{Kg}$, administrados por cinco dias alternados e, diclofenaco ${ }^{7}$ na dose de $50 \mathrm{mg} / \mathrm{kg}$ durante três dias, administrados uma vez ao dia.

Após 16 dias da primeira administração de formaldeído, a epistaxe havia desaparecido. Repetiu-se a endoscopia, observando-se redução acentuada do volume do HEP, localizado apenas na região dos cornetos etmoidais do lado esquerdo e, não mais invadindo o lado direito. A massa residual apresentavase irregular e com áreas de tecido desvitalizado de coloração enegrecida. Após realização de bloqueio anestésico conforme descrito anteriormente, administrou-se $15 \mathrm{~mL}$ de formaldeído a 1,5\%, distribuídos no interior do HEP (Figura 1D). A mesma prescrição de penicilina e diclofenaco já realizada após a primeira sessão de ablação química foi mantida para os dias subsequentes.

Passados 31 dias do segundo tratamento, o exame endoscópico foi repetido revelando que o HEP apresentava aspecto semelhante à avaliação anterior, havia diminuído de volume ainda mais e continuava localizado apenas do lado esquerdo (Figura 1E).

\footnotetext{
${ }^{3}$ Formaldeído solução P.A. - A.C.S. - Labsynth Produtos para Laboratórios Ltda., Diadema - SP

${ }^{4}$ Dormosedan ${ }^{\circledR}$ - Zoetis Inc., Kalamazoo, USA

${ }^{5}$ Xylestesin ${ }^{\circledR} 2 \%$ (sem vasoconstritor) - Cristália

Produtos Químicos e Farmacêuticos Ltda., Itapira - SP

${ }^{6}$ Pentabiótico Reforçado ${ }^{\circledR}$ - Fort Dodge Saúde Animal Ltda., Campinas - SP

${ }^{7}$ Diclofenaco 50 - Ouro Fino Saúde Animal Ltda., Cravinhos - SP
} 


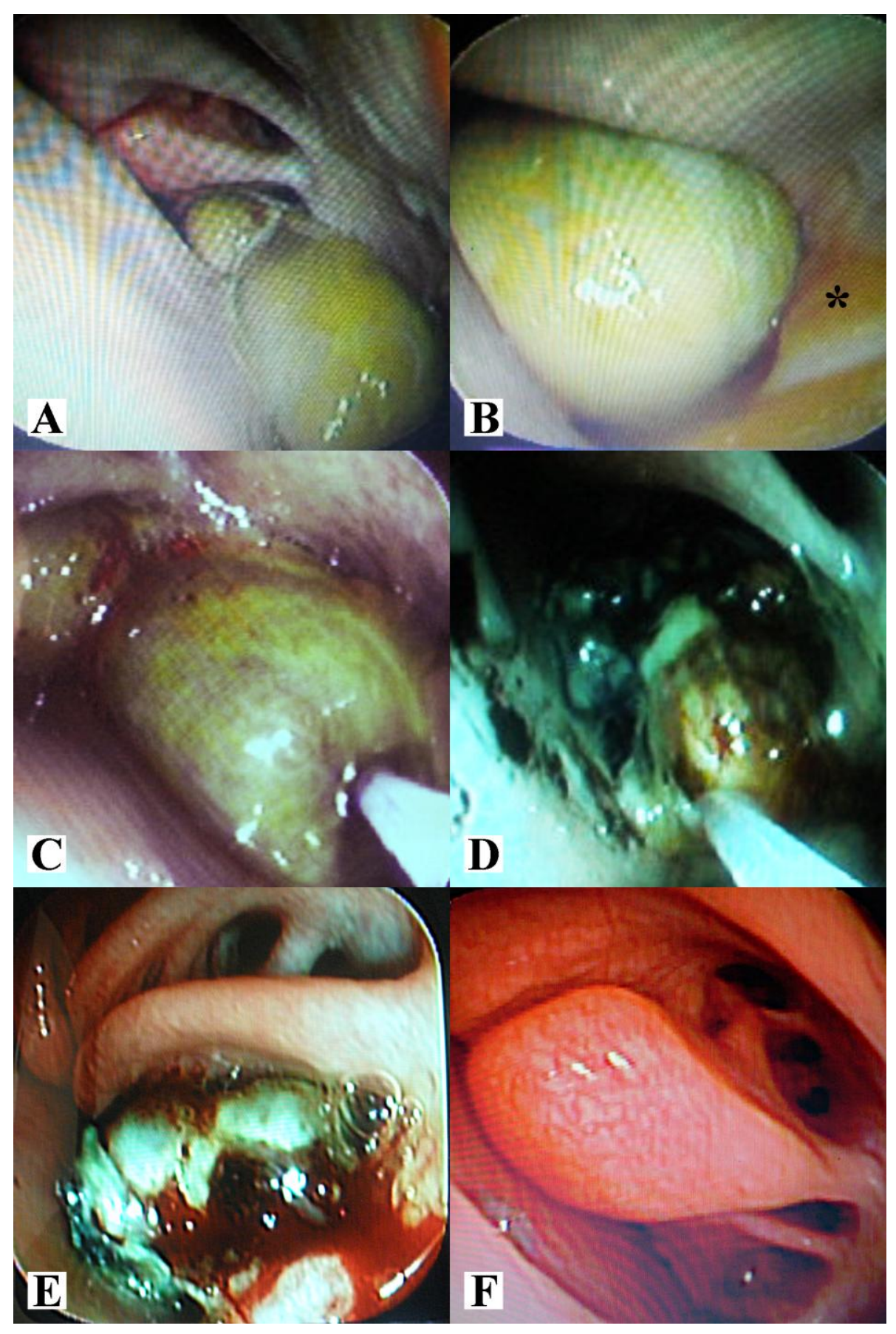

Figura 1 - Imagens endoscópicas da região caudal da cavidade nasal de equino apresentando hematoma etmoidal progressivo (HEP) bilateral. A. Aspecto caudal da cavidade nasal esquerda contendo maior porção do HEP. B. Aspecto caudal da cavidade nasal direita contendo menor porção do HEP, sangue e secreção mucopurulenta (*). C. Primeira administração de solução de formaldeído intralesional para a realização de ablação química do HEP. D. Aspecto do HEP 16 dias após a primeira injeção de formaldeído intralesional, quando realizou-se a segunda etapa do tratamento. E. Aspecto do HEP 31 dias após a segunda injeção de formaldeído intralesional, quando realizou-se a terceira etapa do tratamento. F. Cornetos etmoidais 47 dias após o término do tratamento demonstrando aspecto normal.

Optou-se então pela repetição da ablação química, administrando-se mais $15 \mathrm{~mL}$ de solução de formaldeído a 1,5\% no interior da lesão após o bloqueio anestésico. Administrou-se penicilina e diclofenaco conforme protocolos anteriores. Após a terceira etapa do tratamento, o traqueotubo foi retirado, realizou-se curativo diário da ferida da traqueostomia mediante limpeza com solução de $\mathrm{NaCl}$ a $0,9 \%$ e aplicação de pomada cicatrizante ${ }^{8}$ ao redor, sendo que a mesma cicatrizou por segunda intenção em 15 dias. $\mathrm{O}$ exame endoscópico realizado 47 dias após a terceira administração de formaldeído a 1,5\% revelou ausência

\footnotetext{
${ }^{8}$ Vetaglós Pomada - Vetnil Ind. e Com. de Produtos Veterinários Ltda., Louveira - SP
} 
da lesão (Figura 1F), havendo remissão total dos sinais clínicos. Exames endoscópicos foram realizados mensalmente durante 1 ano, observando-se aspecto normal e ausência de sinais de recidiva.

\section{DISCUSSÃO E CONCLUSÃO}

O presente trabalho descreve um caso de HEP em um equino Puro Sangue Árabe de 13 anos de idade, sendo a faixa etária considerada de maior ocorrência da enfermidade (BELL et al., 1993a). Apenas $15 \%$ a $20 \%$ dos casos de HEP são bilaterais (BOULTON, 1985; GREET, 1992). A apresentação bilateral, com origem em um dos lados e invasão da cavidade nasal contralateral foi descrita por Head \& Dixon (1999) e Stich et al. (2001) e corrobora a presença de epistaxe bilateral. A invasão contralateral só ocorre para os casos em que a origem da massa é nos cornetos etmoidais, portanto classificou-se o presente caso como HEP Tipo 3, de acordo Tate (2002).

A presença de ruído durante a ventilação e dispnéia durante o exercício foi atribuída ao estreitamento da porção caudal das cavidades nasais direita e esquerda (SPECHT et al., 1990; FREEMAN, 2003). O diagnóstico foi confirmado pelo exame endoscópico, identificando-se lesão bilateral de coloração amarelada e superfície lisa e brilhante, de acordo com a descrição clássica de Cook \& Littlewort (1974). Não realizou-se exame radiográfico da cabeça. Apesar do exame radiográfico permitir a identificação de massas etmoidais, sabe-se que devido à superposição de outras estruturas como o globo ocular e a própria porção óssea do labirinto etmoidal, nem sempre a imagem radiográfica permite a identificação da lesão, sendo o exame considerado de valor diagnóstico limitado. Entretanto, tal afirmação vale somente para quando a massa não acomete o interior dos seios paranasais (TREMAINE \& DIXON, 2001). Portanto, como no caso descrito o HEP localizava-se somente na região do etmóide, Tipo 3 segundo Tate (2002), a não realização de exame radiográfico não comprometeu a precisão do diagnóstico.

A confirmação do diagnóstico de HEP só é realizada mediante avaliação histológica de fragmento colhido da lesão via biópsia (GASSER et al., 2000). Entretanto, indica-se que tal procedimento não deve ser realizado em lesões submetidas à ablação química por injeção intralesional de formaldeído (TREMAINE \& DIXON, 2001). Apesar dos autores não relatarem o motivo pelo qual evita-se o procedimento de biópsia, consideramos que tal medida minimizaria o risco hemorragia profusa, uma vez que a solução de formaldeído intralesional causa agressão tecidual, o que poderia dificultar a hemostasia no local de um fragmento que tenha sido retirado. Ainda, sabe-se que se houver regressão do HEP após a ablação química com formaldeído, o diagnóstico está confirmado (SCHUMACHER et al., 1998), pois as lesões consideradas no diagnóstico diferencial, não regrediriam após a terapia em questão.

O tratamento por ablação química com solução de formaldeído intralesional administrado por via endoscópica foi preconizado avaliando-se a dimensão e localização do HEP do equino. Considerou-se eficácia, baixo custo, invasividade mínima e raras complicações, como descrito na literatura (FREES et al., 2001; AHERN \& PARENTE, 2008). Recomenda-se o uso de solução de formaldeído a $4 \%$ que deve ser administrada até preencher a massa e começar a extravasar no ponto de inserção do cateter (SCHUMACHER et al., 1998). Entretanto, devido à extensão e localização bilateral do HEP no caso descrito, preconizou-se a administração de formaldeído na maior porção visível, projetada para a cavidade nasal esquerda. Assim, a massa localizada à direita não foi monitorada por visibilização endoscópica durante a administração e, considerando o risco de extravasamento à direita, utilizou-se solução de formaldeído a 1,5\% visando minimizar lesões de tecidos adjascentes ao HEP, conforme descrito por Frees et al. (2001) com o uso de soluções mais concentradas. Pelo mesmo motivo, limitou-se o volume administrado a $15 \mathrm{ml}$ de solução de formaldeído a $1,5 \%$, ao invés dos $50 \mathrm{ml}$ de solução de formaldeído a $4 \%$ descritos por Freeman (2003).

O tratamento mostrou-se facilmente exequível, pouco invasivo, seguro e de custo reduzido. A epistaxe desapareceu após a primeira injeção de formaldeído a $1,5 \%$ e a completa retração da massa ocorreu após 3 injeções, conforme descrito por Conti et al. (2003) para soluções de formaldeído a $4 \%$. O resultado obtido correspondeu às expectativas do proprietário, com remissão completa dos sinais clínicos, não ocorrendo complicações decorrentes do tratamento instituído.

\section{REFERÊNCIAS}

AHERN, B. J.; PARENTE, E. J. Surgical complications of the equine upper respiratory tract. Veterinary Clinics of North America: Equine Practice, v.24, n.3, p.465-484, 2008.

BELL, B. T. L.; BAKER, G. J.; FOREMAN, J. H. Progressive ethmoid hematoma in horses: background, clinical signs, and diagnosis. Compendium on Continuing Education for the Practicing Veterinarian, v.15, n.8, p.1101-1110, 1993a.

BELL, B. T. L.; BAKER, G. J.; FOREMAN, J. H. Progressive ethmoid hematoma in horses: characteristics, cause, and treatment. Compendium on Continuing Education for the Practicing Veterinarian, v.15, n.10, p.1391-1398, 1993b.

BOULTON, C. H. Equine nasal cavity and paranasal sinus disease: A review of 85 cases. Journal of Equine Veterinary Science, v.5, n.5, p.268-275, 1985.

CONTI, M. B.; MARCHESI, M. C.; RUECA, F.; PUCCETTI, M. Diagnosis and treatment of progressive ethmoidal haematoma (PEH) in horses. Veterinary Research Communications, v.27, n.1, p.739-743, 2003.

COOK, W. R.; LITTLEWORT, M. C. G. Progressive haematoma of the ethmoid region in the horse. Equine Veterinary Journal, v.6, n.3, p.101-108, 1974. 
COLBOURNE, C. M.; ROSENSTEIN, D. S.; STEFICEK, B. A.; YOVICH, J. V.; STICK, J. A. Surgical treatment of progressive ethmoidal hematoma aided by computed tomography in a foal. Journal of the American Veterinary Medical Association, v.211, n.3, p.335-338, 1997.

ETHERINGTON, W. G.; VASEY, J. R.; HORNEY, F. D. Ethmoid hematoma of the equine. Canadian Veterinary Journal, v.23, n.8, p.231-234, 1982.

FEICHTENHOFER, P.; SIMHOFER, H.; HOF, K.; KNEISSL, S. A complementary radiographic projection of the equine maxillary sinus. Journal of Equine Veterinary Science, v.33, n.7, p.565-569.

FREEMAN, D. E. Sinus disease. Veterinary Clinics of North America: Equine Practice, v.19, n.1, p.209243, 2003.

FREES, K. E.; GAUGHAN, E. M.; LILLICH, J. D.; COX, J.; GORONDY, D.; NIETFELD, J. C.; KENNEDY, G. A.; CASH, W. Severe complication after administration of formalin for treatment of progressive ethmoidal hematoma in a horse. Journal of the American Veterinary Medical Association, v.219, n.7, 950-952, 2001.

GASSER, A. M.; LOVE, N. E.; TATE, L. P. Radiographic diagnosis - Ethmoid hematoma. Veterinary Radiology \& Ultrasound, v.41, n.3, p.247249, 2000.

GIBBS, C.; LANE, J. G. Radiographic examination of the facial, nasal and paranasal sinus regions of the horse. Part 2: Radiological findings. Equine Veterinary Journal, v.19, n.5, p.474-482, 1987.

GREET, T. R. C. Outcome of treatment in 23 horses with progressive ethmoidal hematoma. Equine Veterinary Journal, v.24, n.6, p.468-471, 1992.

HEAD, K. W.; DIXON, P. M. Equine nasal and paranasal sinus tumours. Part 1: review of the literature and tumour classification. The Veterinary Journal, v.157, n.3, p.261-278, 1999.

MARRIOTT, M. R.; DART, A. J.; HODGSON, D. R. Treatment of progressive ethmoidal haematoma using intralesional injections of formalin in three horses. Australian Veterinary Journal, v.77, n.6, p.371-373, 1999.

NICKELS, F. A. Nasal passages and paranasal sinuses. In: AUER, J. A.; STICK, J. A. (Eds.) Equine Surgery. 4.ed. St. Louis: Elsevier Saunders, 2012. p.557-568.

NOGUEIRA, G. M.; CATTELAN, J. W.; DUARTE, C. A.; FIALHO, S. S.; MORAES, P. C.; SANTOS, P.S.P. Ablação química de hematoma etmoidal com abordagem auxiliar por sinusostomia frontal - relato de caso. Revista Portuguesa de Ciências Veterinárias, v.102, n.563-564, p.365-368, 2007.
ORSINI, J.A. Chronicle of laser usage in equine surgery. Clinical Techniques in Equine Practice, v.1, n.1, p.3-8, 2002.

ORSINI, J. A.; HAUSNER, E. Presence of vascular endothelial growth factor in progressive equine ethmoid hematoma. International Journal of Applied Research in Veterinary Medicine, v.2, n.3, p.209214, 2004.

PALMER, S. E. The use of lasers for treatment of upper respiratory tract disorders. Veterinary Clinics of North America: Equine Practice, v.19, n.1, p.245263, 2003.

PLATT, H. Haemorrhagic nasal polyps of the horse. Journal of Pathology, v.115, n.1, p.51-55, 1975.

PORTER, E. G.; WERPY, N.M. New concepts in standing advanced diagnostic equine imaging. Veterinary Clinics of North America: Equine Practice, v.30, n.1, p.239-268, 2014.

ROTHAUG, P. G.; TULlENERS, E. P. Neodymium: yttrium-aluminum-garnet laser-assisted excision of progressive ethmoid hematomas in horses: 20 cases (1986-1996). Journal of the American Veterinary Medical Association, v.214, n.7, p.1037-41, 1999.

SCHUMACHER, J.; YARBROUGH, T.; PASCOE, J.; WOODS, P.; MEAGHER, D.; HONNAS, C. Transendoscopic chemical ablation of progressive ethmoidal hematomas in standing horses. Veterinary Surgery, v.27, n.3, p.175-181, 1998.

SOLANO, M.; BRAWER, R. S. CT of the equine head: technical considerations, anatomical guide, and selected diseases. Clinical Techniques in Equine Practice, v.3, n.4, p.374-388, 2004.

SPECHT, T. E.; COLAHAN, P. T.; NIXON, A. J.; BROWN, M. P.; TURNER, T. A.; PEYTON, L. C.; SCHNEIDER, R. K. Ethmoidal hematoma in nine horses. Journal of the American Veterinary Medical Association, v.197, N.5, 613-616, 1990.

STICH, K. L.; RUSH, B. R.; GAUGHAN, E. M. Progressive etmoid hematoma in horses. Compendium on Continuing Education for the Practicing Veterinarian, v.23, n.12, p.1094-1103, 2001.

SULLIVAN, M.; BURRELL, M. H.; McCANDLISH, I. A. Progressive haematoma of the maxillary sinus in a horse. Veterinary Record, v.114, n.8, p.191-192, 1984.

TATE, LP: Application of lasers in equine upper respiratory surgery. Veterinary Clinics of North America: Equine Practice, v.7, n.1, p.165-195, 1991. TATE, L. P. Noncontact free fiber ablation of equine progressive ethmoid hematoma. Clinical Techniques in Equine Practice, v.1, n.1, p.22-27, 2002. 
TEXTOR, J. A.; PUCHALSKI, S. M.; AFFOLTER, V. K.; MacDONALD, M. H.; GALUPPO, L. D.; WISNER, E. R. Results of computed tomography in horses with ethmoid hematoma: 16 cases (1993-2005). Journal of the American Veterinary Medical Association, v.240, n.11, p.1338-1344, 2012.

TREMAINE, W. H.; CLARKE, C. J.; DIXON, P. M. Histopathological findings in equine sinonasal disorders. Equine Veterinary Journal, v.31, n.4, p.296-303, 1999.
TREMAINE, W. H; DIXON, P. M. A long-term study of 277 cases of equine sinonasal disease. Part 1: Details of horses, historical, clinical and ancillary diagnostic findings. Equine Veterinary Journal, v.33, n.3, p.274-282, 2001.

WAGUESPACK, R. W.; TAINTOR, J. Paranasal sinus disease in horses. Compendium on Continuing Education for the Practicing Veterinarian, v.33, n.2, E1-E11, 2011. 